\title{
A Limiter for PPM that Preserves Accuracy at Smooth Extrema
}

\author{
Phillip Colella * \\ Applied Numerical Algorithms Group, Lawrence Berkeley National Laboratory, \\ 1 Cyclotron Road, Berkeley, CA 94720, USA \\ Michael D. Sekora \\ Program in Applied and Computational Mathematics, Princeton University \\ Princeton, NJ 08540, USA
}

\begin{abstract}
We present a new limiter for the PPM method of Colella and Woodward [4] that preserves accuracy at smooth extrema. It is based on constraining the interpolated values at extrema (and only at extrema) using nonlinear combinations of various difference approximations of the second derivatives. Otherwise, we use a standard geometric limiter to preserve monotonicity away from extrema. This leads to a method that has the same accuracy for smooth initial data as the underlying PPM method without limiting, while providing sharp, non-oscillatory representations of discontinuities.
\end{abstract}

Key words: Upwind methods, PPM, limiters.

\section{Introduction}

One of the great successes in numerical methods for hyperbolic conservation laws has been the use of nonlinear hybridization techniques, known as limiters, to maintain positivity and monotonicity in the presence of discontinuities and underresolved gradients. As originally formulated $[3,14,5]$, these methods have the property that the truncation error is first-order accurate at all extrema,

\footnotetext{
* Corresponding author.

Email addresses: pcolella@lbl.gov (Phillip Colella ), sekora@math.princeton. edu (Michael D. Sekora).
}

Preprint submitted to Journal of Computational Physics 14 February 2008 
regardless of the accuracy of the underlying high-order method. This problem has been known since these methods were first introduced, and there have been a variety of methods proposed to deal with it. Typically, these have been based on the idea allowing the representation of solution values outside the range defined by the cell averages [16], while still suppressing oscillations at discontinuities and underresolved gradients. In particular, the methods proposed to solve the problem to obtain uniform high-order accuracy for smooth solutions $[6,8,7,13,2,10]$ typically have used quite elaborate analytic and / or geometric constructions. In this note, we propose a particularly simple approach to solving this problem for the PPM method [4]. It is based on changing the PPM limiter at extrema (and only at extrema) using nonlinear combinations of various difference approximations of the second derivatives. This leads to a method that has the same accuracy for smooth initial data as the underlying PPM method without limiting, while providing sharp, non-oscillatory representations of discontinuities.

\section{Scalar Advection}

We will consider the linear advection equation in one space dimension.

$$
\frac{\partial a}{\partial t}+u \frac{\partial a}{\partial x}=0
$$

We assume that we know at time step $n$ the averages of $a$ over finite volume cells of length $h$.

$$
\langle a\rangle_{j}^{n} \approx \frac{1}{h} \int_{(j-1 / 2) h}^{(j+1 / 2) h} a(x, n \Delta t) d x
$$

The PPM method in [4] for computing $\langle a\rangle_{j}^{n+1}$ is a conservative finite difference method

$$
\langle a\rangle_{j}^{n+1}=\langle a\rangle_{j}^{n}+\frac{u \Delta t}{h}\left(a_{j-\frac{1}{2}}^{n+\frac{1}{2}}-a_{j+\frac{1}{2}}^{n+\frac{1}{2}}\right)
$$

where $a_{j+\frac{1}{2}}^{n+\frac{1}{2}}$ is the average of a parabolic interpolant over the interval swept out by the characteristics crossing the cell face at $\left.\left(j+\frac{1}{2}\right) h\right)$.

$$
\begin{aligned}
a_{j+\frac{1}{2}}^{n+\frac{1}{2}} & =\mathcal{I}_{j,+}(\sigma) \text { if } u>0 \\
& =\mathcal{I}_{j+1,-}(\sigma) \text { otherwise }
\end{aligned}
$$


where $\sigma=|u| \Delta t / h$, and

$$
\begin{aligned}
\mathcal{I}_{j,+}(\sigma) & \equiv \frac{1}{\sigma h} \int_{\left(j+\frac{1}{2}\right) h-\sigma h}^{\left(j+\frac{1}{2}\right) h} a_{j}^{I}(x) d x \\
\mathcal{I}_{j,-}(\sigma) & \equiv \frac{1}{\sigma h} \int_{\left(j-\frac{1}{2}\right) h}^{\left(j-\frac{1}{2}\right) h+\sigma h} a_{j}^{I}(x) d x
\end{aligned}
$$

The parabolic interpolant $a_{j}^{I}(x), x \in[(j-1 / 2) h,(j+1 / 2) h]$ is uniquely determined by the cell average $\langle a\rangle_{j}^{n}$ and the left and right extrapolated edge values $\left.a_{j, \pm}=a_{j}^{I}(j \pm 1 / 2) h\right)$.

$$
\begin{gathered}
a_{j}^{I}(x)=a_{j,-}+\xi\left(a_{j,+}-a_{j,-}+a_{6, j}(1-\xi)\right), a_{6, j}=6\langle a\rangle_{j}^{n}-3\left(a_{j,-}+a_{j,+}\right) \\
\xi=\frac{x-j h}{h}, 0 \leq \xi \leq 1
\end{gathered}
$$

For this choice of interpolant, the averages (5), (6) are given by the following formulas

$$
\begin{aligned}
& \mathcal{I}_{j,+}(\sigma)=a_{j,+}-\frac{\sigma}{2}\left(a_{j,+}-a_{j,-}-\left(1-\frac{2}{3} \sigma\right) a_{6, j}\right) \\
& \mathcal{I}_{j,-}(\sigma)=a_{j,-}+\frac{\sigma}{2}\left(a_{j,+}-a_{j,-}+\left(1-\frac{2}{3} \sigma\right) a_{6, j}\right) .
\end{aligned}
$$

It is easy to check that

$$
\sigma \mathcal{I}_{j,+}(\sigma)+(1-\sigma) \mathcal{I}_{j,-}(1-\sigma)=\langle a\rangle_{j}^{n}, 0 \leq \sigma \leq 1
$$

To complete the description of the algorithm, we must specify how the parabolic interpolant is computed, or, equivalently, how the $a_{j, \pm}$ are computed. In [4], this was done in two steps.

Interpolating face values. We compute high-order accurate approximations to $a$ at cell edges

$$
a_{j+\frac{1}{2}}^{n}=a\left(\left(j+\frac{1}{2}\right) \Delta x, n \Delta t\right)+O\left(h^{p}\right), p \geq 3
$$

and impose the constraint that $a_{j+\frac{1}{2}}^{n}$ must lie between the adjacent cell averages.

$$
\min \left(\langle a\rangle_{j}^{n},\langle a\rangle_{j+1}^{n}\right) \leq a_{j+\frac{1}{2}}^{n} \leq \max \left(\langle a\rangle_{j}^{n},\langle a\rangle_{j+1}^{n}\right)
$$

Constructing the parabolic interpolant. We initialize the left and right extrapolated edge values of the parabolic profile to be $a_{j, \pm}=a_{j \pm \frac{1}{2}}^{n}$, and constrain the left and right edge values so that the interpolated parabolic profile is monotone. 
(1) If $\left(a_{j,+}-\langle a\rangle_{j}^{n}\right)\left(a_{j,-}-\langle a\rangle_{j}^{n}\right)>0$, then we set

$$
a_{j,+}=a_{j,-}=\langle a\rangle_{j}^{n}
$$

(2) Otherwise, if one of $\left|a_{j, \pm}-\langle a\rangle_{j}^{n}\right| \geq 2\left|a_{j, \mp}-\langle a\rangle_{j}^{n}\right|$ then for that choice of $\pm=+,-$ we set

$$
a_{j, \pm}:=\langle a\rangle_{j}^{n}-2\left(a_{j, \mp}-\langle a\rangle_{j}^{n}\right) .
$$

In [4], (12) is computed by taking averages of a piecewise-linear interpolant to which van Leer limiting was applied. In smooth regions away from extrema, this leads to the formula

$$
a_{j+\frac{1}{2}}^{n}=\frac{7}{12}\left(\langle a\rangle_{j}^{n}+\langle a\rangle_{j+1}^{n}\right)-\frac{1}{12}\left(\langle a\rangle_{j-1}^{n}+\langle a\rangle_{j+2}^{n}\right)
$$

which is fourth-order accurate. In addition, the use of van Leer limiters automatically enforces the constraint (13).

For smooth solutions away from from extrema, PPM is third-order accurate for linear advection, and fourth-order accurate in the limit of vanishing CFL number. However, the monotonicity constraints (13), (14), at extrema reduce the truncation error at such locations to $O(h)$. This reduces the overall accuracy of the method, as well as introducing a non-smooth component to the error. To eliminate this problem, we construct a new limiting scheme at extrema to replace (13), (14). It follows the same outline as that given above.

Interpolating face values. We use a linear scheme to compute high-order accurate approximations to $a$ at cell edges, such as the fourth-order discretization (16). To obtain the results presented here, we used the following sixth-order approximation, which provides slightly better accuracy for smooth solutions at low CFL number, at a modest additional cost.

$$
a_{j+\frac{1}{2}}^{n}=\frac{37}{60}\left(\langle a\rangle_{j}^{n}+\langle a\rangle_{j+1}^{n}\right)-\frac{2}{15}\left(\langle a\rangle_{j-1}^{n}+\langle a\rangle_{j+2}^{n}\right)+\frac{1}{60}\left(\langle a\rangle_{j-2}^{n}+\langle a\rangle_{j+3}^{n}\right)
$$

We limit this value by using a nonlinear combination of approximations to the second derivative. If $a_{j+\frac{1}{2}}^{n}$ does not satisfy (13), then we impose the following constraint.

$$
\begin{aligned}
\left(D^{2} a\right)_{j+\frac{1}{2}} & =\frac{3}{h^{2}}\left(\langle a\rangle_{j}^{n}-2 a_{j+\frac{1}{2}}^{n}+\langle a\rangle_{j+1}^{n}\right) \\
\left(D^{2} a\right)_{j+\frac{1}{2}, L} & =\frac{1}{h^{2}}\left(\langle a\rangle_{j-1}^{n}-2\langle a\rangle_{j}^{n}+\langle a\rangle_{j+1}^{n}\right) \\
\left(D^{2} a\right)_{j+\frac{1}{2}, R} & =\frac{1}{h^{2}}\left(\langle a\rangle_{j}^{n}-2\langle a\rangle_{j+1}^{n}+\langle a\rangle_{j+2}^{n}\right)
\end{aligned}
$$

If the signs of $\left(D^{2} a\right)_{j+\frac{1}{2}},\left(D^{2} a\right)_{j+\frac{1}{2},\{L, R\}}$ are all the same, we define

$$
\left(D^{2} a\right)_{j+\frac{1}{2}, l i m}=s \min \left(C\left|\left(D^{2} a\right)_{j+\frac{1}{2}, L}\right|, C\left|\left(D^{2} a\right)_{j+\frac{1}{2}, R}\right|,\left|\left(D^{2} a\right)_{j+\frac{1}{2}}\right|\right)
$$




$$
s=\operatorname{sign}\left(\left(D^{2} a\right)_{j+\frac{1}{2}}\right)
$$

Otherwise, $\left(D^{2} a\right)_{j+\frac{1}{2}, l i m}=0$. Then

$$
a_{j+\frac{1}{2}}^{n}:=\frac{1}{2}\left(\langle a\rangle_{j}^{n}+\langle a\rangle_{j+1}^{n}\right)-\frac{h^{2}}{3}\left(D^{2} a\right)_{j+\frac{1}{2}, l i m}
$$

Here $C>1$ is a constant independent of the mesh spacing $h$. If $a$ is smooth and $\partial_{x}^{2} a \neq 0$ in the neighborhood of the extremum, then $\left(D^{2} a\right)_{j+\frac{1}{2}},\left(D^{2} a\right)_{j+\frac{1}{2}, L}$ and $\left(D^{2} a\right)_{j+\frac{1}{2}, R}$ are all consistent approximations to $\partial_{x}^{2} a$ and differ by no more than $O(h)$ from one another. Thus for $h$ sufficiently small, $\left(D^{2} a\right)_{j+\frac{1}{2}, l i m}=\left(D^{2} a\right)_{j+\frac{1}{2}}$ and $a_{j+\frac{1}{2}}^{n}$ will remain unchanged by the limiting process, thus preserving the accuracy of the interpolated value. We also observe that, at degenerate extrema $\left(\left(\partial_{x} a\right)(x, t)=\left(\partial_{x}^{2} a\right)(x, t)=0\right)$, all three estimates of $\partial_{x}^{2} a$ are themselves no greater than $O\left(h^{2}\right)$, and the resulting value for $a_{j+\frac{1}{2}}^{n}$ is fourth-order accurate. On the other hand, if the violation of the monotonicity constraint (13) is at a face adjacent to a discontinuity, we expect that one of $\left(D^{2} a\right)_{j+\frac{1}{2}, L}$, $\left(D^{2} a\right)_{j+\frac{1}{2}, R}$ will be much smaller in magnitude than $\left(D^{2} a\right)_{j+\frac{1}{2}}$, or have a different sign. In either case, the limiting process will reduce the amplitude of the oscillation, bring the edge value closer to the average of the two adjacent cell averages. This is a simple algebraic form of the mechanism used in [16] by which smooth extrema are left unchanged, while still limiting the interpolated profile at discontinuities.

Constructing the parabolic interpolant. We initialize the left and right extrapolated values $a_{j, \pm}=a_{j \pm 1 / 2}^{n}$, which are then modified to constrain the parabolic profile in each cell. Then the two steps described above for the PPM algorithm are replaced by the following.

(1) If

$$
\begin{gathered}
\left(a_{j,+}-\langle a\rangle_{j}^{n}\right)\left(\langle a\rangle_{j}^{n}-a_{j,-}\right) \leq 0 \\
\text { or } \\
\left(\langle a\rangle_{j-1}^{n}-\langle a\rangle_{j}^{n}\right)\left(\langle a\rangle_{j}^{n}-\langle a\rangle_{j+1}^{n}\right) \leq 0
\end{gathered}
$$

then we are at a local extremum, and we use an approach similar to that used above above to constrain $a_{j \pm \frac{1}{2}}^{n}$ without losing accuracy if the solution is smooth.

$$
\begin{gathered}
\left(D^{2} a\right)_{j}=-\frac{2 a_{6, j}}{h^{2}} \\
\left(D^{2} a\right)_{j, C}=\frac{1}{h^{2}}\left(\langle a\rangle_{j-1}^{n}-2\langle a\rangle_{j}^{n}+\langle a\rangle_{j+1}^{n}\right) \\
\left(D^{2} a\right)_{j, L}=\frac{1}{h^{2}}\left(\langle a\rangle_{j-2}^{n}-2\langle a\rangle_{j-1}^{n}+\langle a\rangle_{j}^{n}\right)
\end{gathered}
$$




$$
\left(D^{2} a\right)_{j, R}=\frac{1}{h^{2}}\left(\langle a\rangle_{j}^{n}-2\langle a\rangle_{j+1}^{n}+\langle a\rangle_{j+2}^{n}\right)
$$

Again, if $\left(D^{2} a\right)_{j},\left(D^{2} a\right)_{j,\{L, C, R\}}$ all have the same sign, then

$$
\begin{gathered}
\left(D^{2} a\right)_{j, l i m}=s \min \left(C\left|\left(D^{2} a\right)_{j, L}\right|, C\left|\left(D^{2} a\right)_{j, R}\right|, C\left|\left(D^{2} a\right)_{j, C}\right|,\left|D^{2} a_{j}\right|\right) \\
s=\operatorname{sign}\left(\left(D^{2} a\right)_{j}\right)
\end{gathered}
$$

for some constant $C>1$, chosen independently from $h$. Otherwise, $\left(D^{2} a\right)_{j, l i m}=0$. Then

$$
a_{j, \pm}:=\langle a\rangle_{j}^{n}+\left(a_{j, \pm}-\langle a\rangle_{j}^{n}\right) \frac{\left(D^{2} a\right)_{j, l i m}}{\left(D^{2} a\right)_{j}}
$$

If the denominator in the last expression vanishes, we set the ratio of the two estimates of $\partial_{x}^{2} a$ to be zero.

The purpose of this limiter is to reduce $a_{6, j}$ so that $\left(D^{2} a\right)_{j}=\left(D^{2} a\right)_{j, l i m}$. Note that if $\left(D^{2} a\right)_{j, l i m}=0$, then the edge values are the same as in (14). As before, at smooth extrema with $\partial_{x}^{2} a \neq 0$, this limiting process leaves the extrapolated edge values unchanged if $h$ is sufficiently small, and introduces an error no greater than $O\left(h^{4}\right)$ at degenerate extrema. Near discontinuities and other underresolved gradients, the same mechanisms for suppressing oscillations as those described for (18), (19) are in effect.

(2) If (20) does not hold, we could proceed as in the original PPM method to impose the constraint (15) to obtain a monotonic parabolic profile in the cell. However, it was pointed out in [15] that the requirement that the parabolic profile be monotone in a cell is more restrictive than is required for the method to be monotonicity-preserving. In the results presented here, we impose a less-restrictive, but sufficient condition analogous to that satisfied by van Leer limiting in [14].

$$
\begin{gathered}
s\langle a\rangle_{j-1}^{n} \leq s \mathcal{I}_{j,-}(\sigma) \leq s\langle a\rangle_{j}^{n} \leq s \mathcal{I}_{j,+}(\sigma) \leq s\langle a\rangle_{j+1}^{n} \\
s=\operatorname{sign}\left(\langle a\rangle_{j+1}^{n}-\langle a\rangle_{j-1}^{n}\right)
\end{gathered}
$$

We define $\alpha_{j, \pm}=a_{j, \pm}-\langle a\rangle_{j}^{n}$. If one of $\left|\alpha_{j, \pm}\right| \geq 2\left|\alpha_{j, \mp}\right|$ holds, then for that choice of $\pm=+$, - we compute

$$
\delta \mathcal{I}_{\text {ext }}=\frac{-\alpha_{j, \pm}^{2}}{4\left(\alpha_{j,+}+\alpha_{j,-}\right)}, \delta a=\langle a\rangle_{j \pm 1}^{n}-\langle a\rangle_{j}^{n}
$$

If $s \delta \mathcal{I}_{\text {ext }} \geq s \delta a$, then we set

$$
a_{j, \pm}:=\langle a\rangle_{j}^{n}-\left(2 \delta a+2 s\left((\delta a)^{2}-\delta a \alpha_{j, \mp}\right)^{\frac{1}{2}}\right)
$$

which causes the parabolic interpolant to satisfy (24). It follows easily from (24) and (11) that

$$
\min \left(\langle a\rangle_{j}^{n},\langle a\rangle_{j-s^{\prime}}^{n}\right) \leq\langle a\rangle_{j}^{n+1} \leq \max \left(\langle a\rangle_{j}^{n},\langle a\rangle_{j-s^{\prime}}^{n}\right), s^{\prime}=\operatorname{sign}(u)
$$


which implies that the scheme is monotonicity-preserving away from extrema.

To summarize, we modify the original PPM algorithm in the following fashion. We use (17) to construct $a_{j+\frac{1}{2}}^{n}$, constraining the values by (18), (19) at locations where (13) is not satisfied. In constructing the parabolic profile, we use (22), (23) to compute the parabolic profile at local extrema where (20) holds. Otherwise, we use a standard monotonicity-preserving limiter such as (15) or (26). In the results presented in the next section, we have used (26).

\section{Results}

We present results for advection in one dimension that demonstrate the improvement of the accuracy of the method using this limiter. We use the following standard 1D test problems [17].

- Gaussian : $a(x, 0)=e^{256\left(x-\frac{1}{2}\right)^{2}}$.

- Semi-circle: $\left.a(x, 0)=\left(\max \left(\frac{1}{16}-\left(x-\frac{1}{2}\right)^{2}\right), 0\right)\right)^{\frac{1}{2}}$

- Square wave: $a(x, 0)=1$ if $\left|x-\frac{1}{2}\right| \leq \frac{1}{4}$, otherwise $a(x, 0)=0$.

All calculations are performed on the unit interval with periodic boundary conditions, advection velocity $u=1$, and CFL number $\sigma=.2$. For the new extremum-preserving limiter, we set the coefficient $C$ appearing in (18), (22) to be $C=1.25$. We have not found any straightforward geometric interpretation of the new limiter to guide our choice of the coefficient $C$, other than it must be greater than 1, independent of $h$. For the test cases presented here, the results appear to be insensitive to the exact value of $C$ over the range 1.25 - 5. In order to obtain third-order accurate results for smooth problems, we initialize the averages of $a$ to be fourth-order accurate at all cells where the solution is smooth.

$$
\langle a\rangle_{j}^{0}=a(j h, 0)+\frac{1}{24}(a((j-1) h, 0)-2 a(j h, 0)+a((j+1) h, 0))
$$

Tables 1 and 2 show errors and rates of convergence for these three test problems using, respectively, the original PPM limiter and the new limiter described here. The use of the extremum-preserving limiter leads to significant improvement in the error in the Gaussian test problem, small improvement in the semi-circle problem, and no significant change in the error for the squarewave problem. The improved accuracy is entirely due to the use of the new limiters that are applied at extrema, and only makes a difference for cases where the error in the old approach is dominated by that at smooth extrema. For problems for which the main error is due to the the presence of discontinuities or other singularities, we expect that the methods should produce very 
similar results. This conclusion is supported by the comparisons in Figures 1 and 2 .

\begin{tabular}{rcccccccc}
\hline$N_{\text {cell }}$ & G $L_{1}$ & Rate & G $L_{\infty}$ & Rate & SC $L_{1}$ & Rate & S $L_{1}$ & Rate \\
\hline 32 & $7.6 \mathrm{E}-2$ & - & $4.8 \mathrm{E}-1$ & - & $7.8 \mathrm{E}-3$ & - & $8.4 \mathrm{E}-2$ & - \\
64 & $2.7 \mathrm{E}-2$ & 1.5 & $2.7 \mathrm{E}-1$ & 0.8 & $4.3 \mathrm{E}-3$ & 0.9 & $4.8 \mathrm{E}-2$ & 0.8 \\
128 & $7.7 \mathrm{E}-3$ & 1.8 & $1.0 \mathrm{E}-1$ & 1.4 & $1.9 \mathrm{E}-3$ & 1.2 & $2.8 \mathrm{E}-2$ & 0.8 \\
256 & $1.3 \mathrm{E}-3$ & 2.6 & $3.1 \mathrm{E}-2$ & 1.7 & $8.3 \mathrm{E}-4$ & 1.2 & $1.6 \mathrm{E}-2$ & 0.8 \\
\hline
\end{tabular}

Table 1: Errors and convergence rates for 1D advection tests for the original PPM Limiter. $\mathrm{G}=$ Gaussian, $\mathrm{SC}=$ semi-circle, $\mathrm{S}=$ square wave. Time

$$
t=10, \sigma=0.2 \text {. }
$$

\begin{tabular}{rcccccccc}
\hline$N_{\text {cell }}$ & G $L_{1}$ & Rate & G $L_{\infty}$ & Rate & SC $L_{1}$ & Rate & S $L_{1}$ & Rate \\
\hline 32 & $4.1 \mathrm{E}-2$ & - & $2.9 \mathrm{E}-1$ & - & $7.3 \mathrm{E}-3$ & - & $7.7 \mathrm{E}-2$ & - \\
64 & $1.1 \mathrm{E}-2$ & 1.9 & $9.7 \mathrm{E}-2$ & 1.6 & $3.2 \mathrm{E}-3$ & 1.2 & $4.4 \mathrm{E}-2$ & 0.8 \\
128 & $2.0 \mathrm{E}-3$ & 2.5 & $1.8 \mathrm{E}-2$ & 2.4 & $1.4 \mathrm{E}-3$ & 1.2 & $2.6 \mathrm{E}-2$ & 0.8 \\
256 & $2.6 \mathrm{E}-4$ & 2.9 & $2.5 \mathrm{E}-3$ & 2.8 & $6.1 \mathrm{E}-4$ & 1.2 & $1.5 \mathrm{E}-2$ & 0.8 \\
\hline
\end{tabular}

Table 2: Errors and convergence rates for 1D advection tests using extremum-preserving limiter. Notation the same as in Table 1.

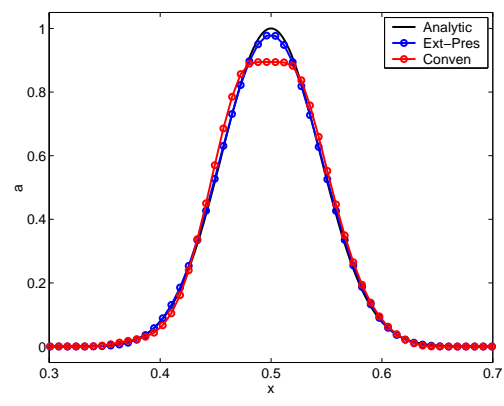

Fig. 1. The extremum-preserving limiter (blue) vs. the original PPM limiter (red) vs. the exact solution (black). Gaussian test, $N_{\text {cell }}=128$, $t=10, \sigma=0.2$.

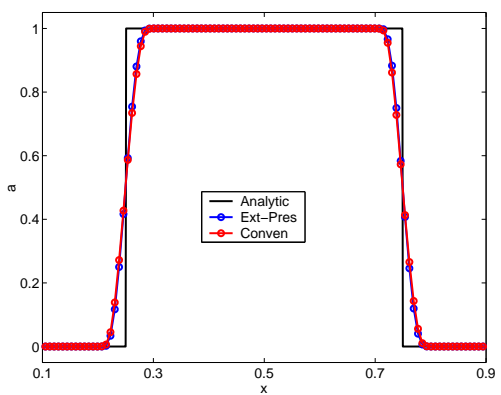

Fig. 2. The extremum-preserving Limiter (blue) vs. the original PPM limiter (red) vs. the exact solution (black). Square-wave test, $N_{\text {cell }}=128, t=10, \sigma=0.2$. 


\section{Conclusions and Future Work}

We presented here a simple method that generalizes the limiter in [4] to preserve the high-order accuracy of the method at smooth extrema. This is done by replacing the constraints (13), (14) at extrema with (19), (23). The impact of the application of this method on the computational cost is negligible, both because of its simplicity, and since it is only applied at extrema. The method described here is only one of a number of design variations using these ideas, some of which are described in [11]. In particular, we can use this approach to obtain a method for computing MUSCL slopes that preserves smooth extrema that is closely related to the approach described in [16]. One can then use this MUSCL calculation as the starting point for computing fourth- or sixth-order edge values, along the lines of the original PPM algorithm.

The extension of PPM to nonlinear systems of hyperbolic conservation laws as described in [4,9] is based the use of an appropriate upstream-centered predictor step that computes (4) for each family of characteristic variables to obtain the left and right states for a Riemann problem, the solution to which is then used to compute the fluxes. The limiter is either applied componentwise to the primitive variables, or applied to the characteristic variables. The method described here can be trivially used for the primitive variable interpolation case, and the construction in [9] can be easily extended to apply it to the characteristic variables; for details, see [11]. Such a method has been implemented in [12] for gas dynamics, and it is observed there that the results obtained using that method eliminate the clipping of smooth extrema, without any loss in robustness of the treatment of strongly nonlinear shocks by the overall method.

This work also provides a starting point for constructing uniformly high-order finite-volume methods for multidimensional hyperbolic PDE, based on highorder quadratures that correctly distinguish between cell averages, face averages, and point values [1], and the method of lines for time discretization. For such an algorithm, we would use the limiter described above to compute (possibly discontinuous) extrapolated edge values, in much the same fashion that the MUSCL limiter is used in method-of-lines calculations.

Finally, there is no guarantee that the method described here preserves global bounds such as positivity. This is an important property for some physical applications such as kinetic systems, where an advected quantity represents a number density or probability density in phase space. To deal with this problem, one can combine the present method with the FCT method in [16], with the latter used only to enforce the global bounds.

Acknowledgements. Work at LBNL was by supported by the U.S. Depart- 
ment of Energy Office of Advanced Scientific Computing Research under contract number DE-AC02-05CH11231. MDS was supported by the U.S. Department of Energy Computational Sciences Graduate Fellowship Program under grant number DE-FG02-97ER25308.

\section{References}

[1] M. Barad and P. Colella. A fourth-order accurate local refinement method for Poisson's equation. Journal of Computational Physics, 209:1-18, 2005.

[2] R. Biswas, K. D. Devine, and J. E. Flaherty. Parallel adaptive finite element methods for conservation laws. Applied Numerical Mathematics, 14:255-284, 1994.

[3] J. P. Boris and D. L. Book. Flux-corrected transport. III. Minimal-error FCT algorithms. Journal of Computational Physics, 20:397-431, 1976.

[4] P. Colella and P. R. Woodward. The Piecewise Parabolic Method (PPM) for gas-dynamical simulations. Journal of Computational Physics, 54:174-201, 1984.

[5] A. Harten. High resolution schemes for hyperbolic conservation laws. Journal of Computational Physics, 49:357-393, 1983.

[6] A. Harten, B. Engquist, S. Osher, and S. R. Chakravarthy. Uniformly high order accurate essentially non-oscillatory schemes, III. Journal of Computational Physics, 77:231-303, 1987.

[7] H. T. Huynh. Accurate upwind methods for the Euler equations. SIAM Journal on Numerical Analysis, 32:1565-1619, 1995.

[8] X. D. Liu, S. Osher, and T. Chan. Weighted essentially non-oscillatory schemes. Journal of Computational Physics, 115:200-212, 1994.

[9] G. H. Miller and P. Colella. A conservative three-dimensional Eulerian method for coupled solid-fluid shock capturing. Journal of Computational Physics, 183:26-82, 2002.

[10] W. J. Rider, J. A. Greenough, and J. R. Kamm. Accurate monotonicity- and extrema-preserving methods through adaptive nonlinear hybridizations. Journal of Computational Physics, 225:1827-1848, 2007.

[11] M. D. Sekora and P. Colella. Extremum-Preserving Limiters for MUSCL and PPM methods. Submitted to arXiv.org, 2008.

[12] J. M. Stone, T. A. Gardiner, P. Teuben, J. F. Hawley, and J. B. Simon. Athena: a new code for astrophysical MHD. Astrophysical Journal Supplement Series, 2008 , in press.

[13] A. Suresh and H. T. Huynh. Accurate monotonicity-preserving schemes with Runge-Kutta time stepping. Journal of Computational Physics 136(1):83-99, 1997. 
[14] B. van Leer. Towards the ultimate conservative difference scheme. IV. A new approach to numerical convection. Journal of Computational Physics, 23:276299, 1977.

[15] P. R. Woodward. Piecewise-parabolic methods for astrophysical fluid dynamics. K. H. A. Winkler and M. L. Norman, editors. Astrophysical Radiation Hydrodynamics, pg 245-326, 1986.

[16] S. T. Zalesak. Fully multidimensional flux-corrected transport algorithms for fluids. Journal of Computational Physics, 31:335-362, 1979.

[17] S. T. Zalesak. Apreliminary comparison of modern shock-capturing schemes: linear advection. In R. Vichnevetsky and R. Stepleman, editors. Advances in Computer Methods for Partial Differential Equations VI p. 15-22, 1987. 